\title{
Interrelationship between Yield and its Contributing Traits in Wheat (Triticum aestivum $\mathrm{L}$ )
}

\author{
Samar Pratap Verma ${ }^{1}$, V.N Pathak ${ }^{1}$ and O.P. Verma ${ }^{2 *}$ \\ ${ }^{1}$ Department of Genetics and Plant Breeding S.M.M. Town PG. College-Ballia, (U.P.), India \\ ${ }^{2}$ Department Of Genetics and Plant Breeding N.D.U.A and T. Kumarganj, \\ Faizabad (U.P.), India \\ *Corresponding author
}

\section{A B S T R A C T}

\section{Keywords}

Wheat, (Triticum aestivum $\mathrm{L})$, CoRelation,

Path coefficient analysis

Article Info

Accepted:

22 January 2019

Available Online:

10 February 2019
A field experiment was conducted to study the character association for 11 yield and its contributing traits in the 93 diverse indigenous genotypes of wheat (including 3 checks) at agricultural research farm Nidharia of S.M.M. Town Post Graduate College, Ballia during Rabi season 2017-2018. The experiment was laid ou tin Augmented Block Design under normal irrigated condition following standard cultural practices. The characters studied were days to $50 \%$ flowering, days to maturity, plant height, tillers per plant, spike length, flag leaf area, peduncle length, 1000-grain weight, biological yield per plant, harvest index and grain yield per plant. The grain yield per plant showed highly significant and positive correlation with tillers per plant, spike length, biological yield per plant, harvest index and test weight. While Flag leaf area and plant height showed nonsignificant and positive correlation with grain yield per plant. The highest positive direct effect on grain yield per plant was observed by biological yield per plant followed by harvest index, days to $50 \%$ flowering, test weight and tillers per plant while remaining traits showed negative direct effect on grain yield per plant. Hence, for the development of high yielding wheat varieties these traits possessing highly significant positive associations should be given more weightage in breeding or selection program.

\section{Introduction}

Wheat, (Triticum aestivum L) world's largest self- pollinated crop of the cereal crop which belongs to Gramineae (Poaceae) family of the genus Triticum. It has been described as the 'King of cereals'. Wheat is consumed in a variety of ways such as bread chapatti, porridge (Daliya), flour, etc. It is grown in diversified environments. India is the second largest producer of wheat after China. India's share in world, wheat production is about $14.65 \%$ of world's wheat production. Wheat may be compared well with other cereals in nutritive value. There is much scope to breed wheat varieties for higher yield coupled with acceptable quality. It has good nutrition profile with $12.1 \%$ protein, $1.8 \%$ lipids, $1.8 \%$ 
ash, $2.0 \%$ reducing sugars, $6.7 \%$ pentose, $59.2 \%$ starch, $70 \%$ total carbohydrates and provides $314 \mathrm{~K} \mathrm{cal} / 100 \mathrm{~g}$ of food. It is also a good source of minerals and vitamins viz., calcium (37 mg/100g), iron (4.1 mg/100g), thiamine $\quad(0.45 \mathrm{mg} / 100 \mathrm{~g}), \quad$ riboflavin $(0.13 \mathrm{mg} / 100 \mathrm{~g})$ and nicotinic acid (5.4mg/100mg). During genetic improvement of yield in crop plants selection and hybridization techniques are utilized frequently. Selection is usually practiced for pooling favorable genes, while hybridization is predominantly utilized to accumulate favorable genes in a variety for obtaining better performance. Yield being a complex character is a function of several component characters and their interaction with environment. Genotypic and phenotypic correlation reveals the degree of association between different characters and thus aid in selection to improve the yield and yield attributing characters simultaneously. Further, path coefficient analysis helps in partitioning of correlation coefficients into direct and indirect effects and in the assessment of relative contribution of each component character to the yield.

\section{Material and Methods}

The experiment of present investigation was conducted to evaluate the ninety three wheat germplasm lines including three checks (namely HD 2967, HI 8713, and SONALIKA) in Augmented Block Design at Agricultural Research Farm, Nidharia, S.M.M. Town Post Graduate College, Ballia (U.P.). These genotypes exhibited wide spectrum of variation for various agronomical and morphological characters. The experimental field was divided into 9 blocks and 13 plots in each block (10 test genotypes along with 3 checks) was accommodated in each block. Each plot was consist of two rows of $2.5 \mathrm{~m}$ length with spacing of $5 \mathrm{~cm}$ within the rows and $25 \mathrm{~cm}$ between the rows. The recommended cultural practices were followed to raise a good normal crop. The observations were recorded on ten randomly selected plants from each plot except days to $50 \%$ flowering and days to maturity. Ten competitive plants from each plot were randomly selected for recording observations for all the quantitative characters except days to flowering and maturity, which was recorded on the plot basis. The data were recorded for the following characters; days to $50 \%$ flowering, days to maturity, plant height $(\mathrm{cm})$, tillers per plant, spike length $(\mathrm{cm})$, peduncle length $(\mathrm{cm})$, flag leaf area $\left(\mathrm{cm}^{2}\right)$, biological yield per plant $(\mathrm{g})$, harvest index (\%), test weight (1000-grain weight) (g) and grain yield per plant (g). Statistical analysis was carried out according to standard statically procedure (Federer, 1956). The simple correlations between different characters were estimated according to Searle (1961) while path coefficient analysis following Dewey and Lu (1959) to examine genetic interrelationships in existing divers wheat genotypes.

\section{Results and Discussion}

The analysis of variance revealed highly significant differs between the genotypes. The grain yield or economic yield, in almost all the crops, is the complex character which manifests from multiplicative interactions of several other characters that are termed as yield components. The correlation coefficient is the measure of degree of symmetrical association between two variables or characters which helps us in understanding the nature and magnitude of association among yield and yield components.

In the present investigation, simple correlation coefficients were computed among 11 characters (Table 1). The grain yield per plant exhibited highly significant and positive association with tillers per plant, spike length, biological yield per plant, harvest index and 
test weight. Thus, the high yielding wheat genotypes are likely to possess higher biological yield per plant, spike length, tillers per plant, harvest index and test weight along with undesirable tall stature, late flowering and late maturity. The strong positive associations of grain yield with characters mentioned above have also been reported earlier in wheat (Arya et al., 2005; Yadavet al., 2006; Singh et al., 2008; Bisht and Gahalian. 2009, Singh et al., 2010; Kumar et al., 2014 and Phougat et al., 2017). The biological yield per plant, having highest positive correlation with flag leaf area, tillers per plant and spike length, but it had negative association with days to $50 \%$ flowering and days to maturity. The strong positive association of biological yield per plant with the characters like flag leaf area, tillers per plant and spike length which have increasing effect on over all biomass production appears logical.

Harvest index exhibited significant and negative association with biological yield per plant, flag leaf area and peduncle length. Similarly, 1000-grain weight recorded highly significant and positive nature with harvest index and plan height. The above discussion revealed that wheat genotypes with higher grain yield and biomass production potential had higher mean performance for tillers per plant, spike length, biological yield per plant, harvest index, test weight and flag leaf area.

Spike length showed highly significant and positive correlation with peduncle length, biological yield per plant and grain yield per plant. Flag leaf area showed highly significant and positive correlation with spike length and biological yield per plant, and it's also showed significant and positive correlation with plant height and peduncle length. This suggested that the genotypes having large flag leaf area had tall plant height, tall spike length, tall peduncle length and highest biological yield.
Path coefficient analysis is a tool to partition the observed correlation coefficient into direct and indirect effects of yield components on grain yield. Path analysis provides clearer picture of character associations for formulating efficient selection strategy. Path coefficient analysis differs from simple correlation in that it points out the causes and their relative importance, whereas, the later measures simply the mutual association ignoring the causation. The results of path coefficient analysis carried out using simple correlation coefficients among 11 characters are given in Table 2. Biological yield per plant followed by harvest index, showed very high positive direct contribution on grain yield per plant. Thus, biological yield per plant and harvest index emerged as major direct yield components.

The available literature has also identified biological yield per plant and harvest index as important direct contributors to grain yield per plant (Singh et al., 2012; Kumar et al., 2010; Phougat et al., 2017). The direct effects of remaining eight characters viz., days to $50 \%$ flowering, days to maturity, flag leaf area, plant height and peduncle length were very low and non-significant indicating their negligible direct contribution towards grain yield.

The highest indirect effects of tillers per plant, spike length and flag leaf area possessed high order positive estimates via biological yield per plant on grain yield per plant. Thus, the above four characters emerged as most important indirect yield contributing characters because they showed substantial positive indirect effects on grain yield per plant via biological yield per plant. The three characters mentioned above have also been found as important contributors to grain yield in wheat as reported by earlier workers (Singh et al., 2010; Anwar et al., 2009; Tripathi et al., 2011). 
Table.1 Estimates of simple correlation coefficients between 11 characters in wheat

\begin{tabular}{|c|c|c|c|c|c|c|c|c|c|c|c|}
\hline Character & $\begin{array}{l}\text { Days to } \\
50 \% \\
\text { flowering }\end{array}$ & $\begin{array}{l}\text { Flag leaf } \\
\text { area } \\
\left(\mathrm{cm}^{2}\right)\end{array}$ & $\begin{array}{l}\text { Plant } \\
\text { height } \\
(\mathrm{cm})\end{array}$ & $\begin{array}{l}\text { Days to } \\
\text { maturity }\end{array}$ & $\begin{array}{l}\text { Tillers/ } \\
\text { plant }\end{array}$ & $\begin{array}{l}\text { Spike } \\
\text { length } \\
(\mathrm{cm})\end{array}$ & $\begin{array}{l}\text { Peduncle } \\
\text { length } \\
(\mathrm{cm})\end{array}$ & $\begin{array}{l}\text { Biological } \\
\text { yield/plant } \\
\text { (g) }\end{array}$ & $\begin{array}{l}\text { Harvest } \\
\text { index }(\%)\end{array}$ & $\begin{array}{l}\text { Test } \\
\text { weight (g) }\end{array}$ & $\begin{array}{l}\text { Grain } \\
\text { yield/plant } \\
\text { (g) }\end{array}$ \\
\hline $\begin{array}{l}\text { Days to } \\
\mathbf{5 0 \%} \\
\text { Flowering }\end{array}$ & $\begin{array}{l}\mathbf{r}(\mathbf{g}) \\
\mathbf{r}(\mathbf{p})\end{array}$ & $\begin{array}{l}-0.2501 \\
-\mathbf{0 . 2 2 3 5} *\end{array}$ & $\begin{array}{l}-0.1336 \\
\mathbf{- 0 . 1 1 9 9}\end{array}$ & $\begin{array}{l}0.8691 \\
\mathbf{0 . 8 0 1 7} * *\end{array}$ & $\begin{array}{l}0.0424 \\
\mathbf{0 . 0 1 9 7}\end{array}$ & $\begin{array}{l}-0.2362 \\
-\mathbf{0 . 2 0 3 2} *\end{array}$ & $\begin{array}{l}-0.1564 \\
-\mathbf{0 . 1 0 9 6}\end{array}$ & $\begin{array}{l}-0.0651 \\
\mathbf{- 0 . 0 6 5 7}\end{array}$ & $\begin{array}{l}-0.0948 \\
-\mathbf{0 . 0 6 5 7}\end{array}$ & $\begin{array}{l}-0.2211 \\
-0.2136 *\end{array}$ & $\begin{array}{l}-0.1156 \\
\mathbf{- 0 . 1 0 1 6}\end{array}$ \\
\hline $\begin{array}{l}\text { flag leaf area } \\
\left(\mathrm{cm}^{2}\right)\end{array}$ & & $\begin{array}{l}\mathbf{r}(\mathbf{g}) \\
\mathbf{r}(\mathbf{p})\end{array}$ & $\begin{array}{l}0.1871 \\
\text { 0.1991* }\end{array}$ & $\begin{array}{l}-0.1747 \\
-\mathbf{0 . 1 6 7 9}\end{array}$ & $\begin{array}{l}-0.2193 \\
\mathbf{- 0 . 1 2 0 4}\end{array}$ & $\begin{array}{l}0.3485 \\
\mathbf{0 . 3 0 9 1} * *\end{array}$ & $\begin{array}{l}0.2627 \\
\mathbf{0 . 2 1 1 1} *\end{array}$ & $\begin{array}{l}0.2032 \\
\mathbf{0 . 2 5 4 1} * *\end{array}$ & $\begin{array}{l}-0.2334 \\
\mathbf{- 0 . 1 9 7 1} *\end{array}$ & $\begin{array}{l}0.0942 \\
\mathbf{0 . 1 1 9 2}\end{array}$ & $\begin{array}{l}0.0333 \\
\mathbf{0 . 0 9 2 6}\end{array}$ \\
\hline $\begin{array}{l}\text { Plant } \\
\text { height }(\mathrm{cm})\end{array}$ & & & $\begin{array}{l}\mathbf{r}(\mathbf{g}) \\
\mathbf{r}(\mathbf{p})\end{array}$ & $\begin{array}{l}0.0199 \\
\mathbf{0 . 0 2 9 1}\end{array}$ & $\begin{array}{l}-0.1586 \\
-\mathbf{0 . 1 1 0 0}\end{array}$ & $\begin{array}{l}0.0724 \\
\mathbf{0 . 0 6 6 5}\end{array}$ & $\begin{array}{l}0.1085 \\
\mathbf{0 . 1 0 2 2}\end{array}$ & $\begin{array}{l}0.0118 \\
\mathbf{0 . 0 3 3 3}\end{array}$ & $\begin{array}{l}0.0802 \\
\mathbf{0 . 0 5 4 1}\end{array}$ & $\begin{array}{l}0.1874 \\
0.1854 * *\end{array}$ & $\begin{array}{l}0.0588 \\
\mathbf{0 . 0 6 1 5}\end{array}$ \\
\hline $\begin{array}{l}\text { Days to } \\
\text { Maturity }\end{array}$ & & & & $\begin{array}{l}\mathbf{r}(\mathbf{g}) \\
\mathbf{r}(\mathbf{p})\end{array}$ & $\begin{array}{l}-0.0441 \\
\mathbf{- 0 . 0 8 1 6}\end{array}$ & $\begin{array}{l}-0.2209 \\
-\mathbf{0 . 1 7 8 9}\end{array}$ & $\begin{array}{l}-0.0258 \\
\mathbf{- 0 . 0 3 4 1}\end{array}$ & $\begin{array}{l}-0.1823 \\
\mathbf{- 0 . 1 3 9 6}\end{array}$ & $\begin{array}{l}0.0802 \\
\mathbf{0 . 0 5 7 2}\end{array}$ & $\begin{array}{l}-0.1825 \\
-\mathbf{0 . 1 5 8 8}\end{array}$ & $\begin{array}{l}-0.1958 \\
-\mathbf{0 . 1 1 9 3}\end{array}$ \\
\hline $\begin{array}{l}\text { Tillers/ } \\
\text { Plant }\end{array}$ & & & & & $\begin{array}{l}\mathbf{r}(\mathbf{g}) \\
\mathbf{r}(\mathbf{p})\end{array}$ & $\begin{array}{l}-0.0215 \\
-\mathbf{0 . 0 0 7 2}\end{array}$ & $\begin{array}{l}-0.2825 \\
-\mathbf{0 . 2 0 9 3} *\end{array}$ & $\begin{array}{l}0.4608 \\
0.4248 * *\end{array}$ & $\begin{array}{l}0.0935 \\
\mathbf{0 . 0 4 3 4}\end{array}$ & $\begin{array}{l}-0.1445 \\
-\mathbf{0 . 1 0 5 6}\end{array}$ & $\begin{array}{l}0.4892 \\
\mathbf{0 . 4 2 6 5} * *\end{array}$ \\
\hline $\begin{array}{l}\text { Spike length } \\
\text { (cm) }\end{array}$ & & & & & & $\begin{array}{l}\mathbf{r}(\mathbf{g}) \\
\mathbf{r}(\mathbf{p})\end{array}$ & $\begin{array}{l}0.3285 \\
\mathbf{0 . 3 1 7 8} * *\end{array}$ & $\begin{array}{l}0.3534 \\
0.3099 * *\end{array}$ & $\begin{array}{l}0.0184 \\
\mathbf{0 . 0 1 8 0}\end{array}$ & $\begin{array}{l}0.0943 \\
\mathbf{0 . 0 8 8 0}\end{array}$ & $\begin{array}{l}0.3198 \\
\mathbf{0 . 2 7 5 7 * *}\end{array}$ \\
\hline $\begin{array}{l}\text { Peduncle } \\
\text { length }(\mathrm{cm})\end{array}$ & & & & & & & $\begin{array}{l}\mathbf{r}(\mathbf{g}) \\
\mathbf{r}(\mathbf{p})\end{array}$ & $\begin{array}{l}0.2185 \\
\mathbf{0 . 1 2 8 3}\end{array}$ & $\begin{array}{l}-0.2690 \\
-\mathbf{0 . 2 2 1 3}\end{array}$ & $\begin{array}{l}0.1792 \\
\mathbf{0 . 1 4 9 9}\end{array}$ & $\begin{array}{l}0.0221 \\
-\mathbf{0 . 0 2 6 8}\end{array}$ \\
\hline $\begin{array}{l}\text { Biological } \\
\text { yield/plant (g) }\end{array}$ & & & & & & & & $\begin{array}{l}\mathbf{r}(\mathbf{g}) \\
\mathbf{r}(\mathbf{p})\end{array}$ & $\begin{array}{l}-0.2661 \\
\mathbf{- 0 . 2 0 9 3} *\end{array}$ & $\begin{array}{l}0.1437 \\
\mathbf{0 . 1 7 8 4}\end{array}$ & $\begin{array}{l}0.7910 \\
\mathbf{0 . 7 7 9 1} * *\end{array}$ \\
\hline $\begin{array}{l}\text { Harvest index } \\
(\%)\end{array}$ & & & & & & & & & $\begin{array}{l}\mathbf{r}(\mathbf{g}) \\
\mathbf{r}(\mathbf{p})\end{array}$ & $\begin{array}{l}0.4257 \\
\mathbf{0 . 3 9 3 7} * *\end{array}$ & $\begin{array}{l}0.3054 \\
\mathbf{0 . 3 9 2 1} * *\end{array}$ \\
\hline $\begin{array}{l}\text { Test weight } \\
\text { (g) }\end{array}$ & & & & & & & & & & $\begin{array}{l}\mathbf{r}(\mathbf{g}) \\
\mathbf{r}(\mathbf{p})\end{array}$ & $\begin{array}{l}0.3999 \\
\mathbf{0 . 4 0 9 3} * *\end{array}$ \\
\hline $\begin{array}{l}\text { Grain } \\
\text { yield/plant (g) }\end{array}$ & & & & & & & & & & & $\begin{array}{l}\mathbf{r}(\mathbf{g}) \\
\mathbf{r}(\mathbf{p})\end{array}$ \\
\hline
\end{tabular}


Table.2 Direct and indirect effects of 10 characters on grain yield per plant in wheat

\begin{tabular}{|c|c|c|c|c|c|c|c|c|c|c|c|}
\hline Character & $\begin{array}{c}\text { Days to } \\
50 \% \text { flowering }\end{array}$ & $\begin{array}{l}\text { Flag } \\
\text { leaf } \\
\text { area } \\
\left(\mathrm{cm}^{2}\right)\end{array}$ & $\begin{array}{l}\text { Plant } \\
\text { height } \\
(\mathrm{cm})\end{array}$ & $\begin{array}{l}\text { Days to } \\
\text { maturity }\end{array}$ & $\begin{array}{c}\text { Tillers } \\
\text { plant }\end{array}$ & $\begin{array}{l}\text { Spike } \\
\text { length } \\
\text { (cm) }\end{array}$ & $\begin{array}{l}\text { Peduncle } \\
\text { length } \\
\text { (cm) }\end{array}$ & $\begin{array}{c}\text { Biological } \\
\text { yield/plant } \\
\text { (g) }\end{array}$ & $\begin{array}{l}\text { Harvest } \\
\text { index } \\
(\%)\end{array}$ & $\begin{array}{c}\text { Test } \\
\text { weight } \\
\text { (g) }\end{array}$ & $\begin{array}{l}\text { Grain } \\
\text { yield/ } \\
\text { plant (g) }\end{array}$ \\
\hline $\begin{array}{c}\text { Days to } \\
50 \% \\
\text { flowering }\end{array}$ & 0.0436 & $\begin{array}{c}- \\
0.0097\end{array}$ & 0.0052 & 0.0350 & 0.0009 & -0.0089 & -0.0048 & -0.0029 & -0.0029 & $\begin{array}{c}- \\
0.0093\end{array}$ & -0.1016 \\
\hline $\begin{array}{c}\text { Flag leaf } \\
\text { area } \\
\left(\mathrm{cm}^{2}\right)\end{array}$ & 0.0049 & $\begin{array}{c}- \\
0.0221\end{array}$ & $\begin{array}{c}- \\
0.0044\end{array}$ & 0.0037 & 0.0027 & -00068 & -0.0047 & -0.0056 & 0.0043 & $\begin{array}{c}- \\
0.0026\end{array}$ & 0.0926 \\
\hline $\begin{array}{c}\text { Plant } \\
\text { height }(\mathrm{cm})\end{array}$ & -0.0012 & 0.0020 & 0.0101 & 0.0003 & $\begin{array}{c}- \\
0.0011\end{array}$ & 0.0007 & 0.0010 & 0.0003 & 0.0005 & 0.0019 & 0.0615 \\
\hline $\begin{array}{l}\text { Days to } \\
\text { Maturity }\end{array}$ & -0.0488 & 0.0102 & $\begin{array}{c}- \\
0.0018\end{array}$ & -0.0608 & 0.0050 & 0.0109 & 0.0021 & 0.0085 & -0.0035 & 0.0097 & -0.1193 \\
\hline $\begin{array}{l}\text { Tillers/ } \\
\text { Plant }\end{array}$ & 0.0004 & $\begin{array}{c}- \\
0.0025 \\
\end{array}$ & $\begin{array}{c}- \\
0.0023 \\
\end{array}$ & -0.0017 & 0.0211 & -0.0002 & -0.0044 & 0.0090 & 0.0009 & $\begin{array}{c}- \\
0.0022 \\
\end{array}$ & $0.4265^{* *}$ \\
\hline $\begin{array}{c}\text { Spike } \\
\text { length }(\mathrm{cm})\end{array}$ & 0.0009 & $\begin{array}{c}- \\
0.0013\end{array}$ & $\begin{array}{c}- \\
0.0003\end{array}$ & 0.0008 & 0.0000 & -0.0042 & -0.0013 & -0.0013 & -0.0001 & $\begin{array}{c}- \\
0.0004\end{array}$ & $0.2757 * *$ \\
\hline $\begin{array}{c}\text { Peduncle } \\
\text { length }(\mathrm{cm})\end{array}$ & 0.0010 & $\begin{array}{c}- \\
0.0019 \\
\end{array}$ & $\begin{array}{c}- \\
0.0009 \\
\end{array}$ & 0.0003 & 0.0019 & -0.0028 & -0.0089 & -0.0011 & 0.0020 & $\begin{array}{c}- \\
0.0013 \\
\end{array}$ & -0.0268 \\
\hline $\begin{array}{c}\text { Biological } \\
\text { yield/plant } \\
(\mathrm{g})\end{array}$ & -0.0581 & 0.2245 & 0.0295 & -0.1234 & 0.3754 & 0.2739 & 0.1133 & $\mathbf{0 . 8 8 3 7}$ & -0.1850 & 0.1576 & $0.7791 * *$ \\
\hline $\begin{array}{c}\text { Harvest } \\
\text { index }(\%)\end{array}$ & -0.0369 & $\begin{array}{c}- \\
0.1108 \\
\end{array}$ & 0.0304 & 0.0321 & 0.0244 & 0.0101 & -0.1244 & -0.1176 & 0.5620 & 0.2213 & $0.3921 * *$ \\
\hline $\begin{array}{l}\text { Test weight } \\
\text { (g) }\end{array}$ & -0.0074 & 0.0041 & 0.0064 & -0.0055 & $\begin{array}{c}- \\
0.0037 \\
\end{array}$ & 0.0031 & 0.0052 & 0.0062 & 0.0137 & 0.0347 & $04093 * *$ \\
\hline
\end{tabular}

Residual factor $=0.2597$

Bold figures indicate direct effects. 
In contrast, tillers per plant and spike length exerted high order positive indirect effect via biological yield per plant and substantial negative indirect effects via harvest index on grain yield per plant. Similarly, 1000-grain weight showed high order positive indirect effect via harvest index. The biological yield per plant, which had highest positive direct effect on grain yield per plant, also exerted considerable negative indirect effect on grain yield per plant via harvest index. Thus, characters like tillers per plant, spike length, 1000-grain weight, harvest index and biological yield per plant may also be considered as important indirect yield components of complex nature due to their contrasting positive and negative indirect effects via one or other characters. Thus, these five characters need special attention at the time of formulation of selection strategy due to their contrasting direct and indirect effects. The occurrence of contrasting positive and negative direct/indirect effect by same character via one or another characters as observed in present study is in conformity with earlier reports of (Sachan and singh, 2003; Muhammad and Ihsan, 2004) Majority of the estimates of direct and indirect effects were too low to be considered of any consequence. This may be attributed to presence of very high genetic variability and diversity in the fairly large number of germplasm lines. The existence of different character combinations in diverse germplasm lines might have led to different types of character associations in different lines. Thus, presence of several contrasting types of character associations and inter relationships might have resulted into cancellation of contrasting associations by each other ultimately leading to lowering of the few estimates of certain traits.

In the present study, path analysis reflected that biological yield per plant, harvest-index and test weight as important direct yield contributing characters. On the other handtillers per plant, spike length, flag leaf area and test weight emerged as most important indirect yield components, while days to $50 \%$ flowering, days to maturity, 1000-grain weight, harvest index and biological yield appeared as important but complex indirect yield contributors due to their contrasting positive and negative indirect effect via different characters. These findings are in close association with the results of Subhani, 2000; Singh et al., 2010; It reflex that emphasis should be given to select the above mentioned traits to enhance the production and productivity of wheat under irrigated conditions in northern- eastern plane zone.

\section{References}

Analysis for some metric traits in wheat. International J. Agric. Biology, Pakistan. 6 (1): 138-142.

Anwar, J., Ali, M. A., Hussain, M., Sabir, W., Khan, M. A., Zulkiffal, M. and Abdullah, M. (2009). Assessment of yield criteria in bread wheat through correlation and path analysis. JAPS, J. Animal and Plant Sci. 19 (4): 185-188.

Arya, V. D.; Pawar, I. S.; Lamba, R. A. S. (2005).Genetic variability, correlation and path analysis for yield and quality traits in bread wheat. Haryana Agricultural University Journal of Research. 35(1):59-63. 7

Bisht and Gahalain, S. S (2009). Interrelationships and path coefficient analysis in wheat germplasm of Kumaun Himalayas. Vegetos. 22 (2): 19-26.

Dewey, D.R. and Lu, K.H. 1959. A correlation and path analysis of components of crested wheat grass seed production. Agron. J. 57: 515518.

Federer, W. T. 1956.Augmented Designs. Hawaii Planters' Record LV. (2): 191208. 
Kumar, H., Khosla, G. and Sharma, P. K. (2010). Utilization of genetic variability, correlation and path analysis for seed yield improvement in bread wheat (Triticum aestivum L.) genotypes. Environment and Ecology. 28(1): 91-94.

Kumar, Y., Lamba, R. A. S.; Balbir Singh; Vinod Kumar. (2014). Genetic variability, correlation and path analysis in wheat varieties under late sown condition. Annals of Agri Bio Research. 19(4):724-727.

Muhamad, K. And Ihsan, K. 2004. Heritability, correlation and pathcoefficient

Phougat Divya; Panwar, I. S., Saharan, R. P., Vikram Singh; AnuradhaGodara. (2017). Genetic diversity and association studies for yield attributing traits in bread wheat [Triticum aestivum (L.) em.Thell]. Research on Crops. 18 (1): 139-144.

Sachan, M.S. And Singh, S.P., 2003. Genetics of yield and its components in durum wheat (T. durum Desf.). Journal of Inter Academicia. 7 (2): 140- 143.

Searle, S.R. 1961. Phenotypic, genotypic and environmental correlations. Biometrics. 17: 474-480.

Singh, A. K., Singh, S. B., Singh, A. P., Sharma, A. K. (2012). Genetic variability, character association and path analysis for seed yield and its component characters in wheat (Triticum aestivum L.) under rainfed environment. Indian Journal of Agricultural Research. 46(1): 48-53.

Singh, B. N., Vishwakarma, S. R. and Singh, V. K. (2010). Character association and path analysis in elite lines of wheat (Triticum aestivum L.). Plant Archives, 10 (2): 845-847

Singh, S. K., Singh, B. N., Singh, P. K., Sharma, C. L. (2008). Correlation and path analysis in some exotic lines in wheat (Triticum aestivumL.). New Botanist. 35(1/4): 89-94

Subhani, G.M., 2000. Correlation and pathcoefficient analysis in bread wheat

Tripathi, G.P. Parde, N.S. Zate, D.K. Lal, G.M. (2014) Genetic variability and heritability studies on bread wheat (Triticum aestivum L.). International Journal of Plant Sciences, 10(1):57-59.

Under drought stress and normal conditions. Pakistan J. Biological Sci. 3(1): 72-77.

Yadav, D. K., Pawar, I. S., Sharma, G. R., Lamba, R. A. S. (2006). Evaluation of variability parameters and path analysis in bread wheat. National Journal of Plant Improvement. 8(1): 86-89.

\section{How to cite this article:}

Samar Pratap Verma, V.N Pathak and Verma, O.P. 2019. Interrelationship between Yield and its Contributing Traits in Wheat (Triticum aestivum L.) Int.J.Curr.Microbiol.App.Sci. 8(02): 3209-3215. doi: https://doi.org/10.20546/ijcmas.2019.802.375 